\title{
A Comparative Study of Call Admission Control in Mobile Multimedia Networks using Soft Computing
}

\author{
Sanjeev Kumar \\ Department of Computer Science \\ Grukul Kangri University \\ Haridwar, UK, India
}

\author{
Krishan Kumar \\ Department of Computer Science \\ Grukul Kangri University \\ Haridwar, UK, India
}

\author{
Anand Kumar Pandey \\ School of Electronics Engg. \\ Shobhit University \\ Meerut, UP, India
}

\begin{abstract}
Call admission control (CAC) is one of challenging issues for quality of service $(\mathrm{QoS})$ provisioning in mobile multimedia network communication systems. CAC can be defined as the system that restricts the network access based on resource availability. A new call is admitted if enough idle resources are available in order to meet the QoS requirements of the new call without violating the QoS for already accepted calls, otherwise the call is rejected. This paper provides a survey of different CAC schemes used for mobile multimedia networks using soft computing techniques like artificial neural networks, fuzzy logic, and genetic algorithms. Soft computing can be used to obtain optimized results from many parameters used in mobile multimedia networks. Our goal is to review and compare the best features of soft computing applied in mobile multimedia networks to improve the capability of existing CAC methods. This paper also elaborates the future directions for the use of untouched areas of artificial neural networks, fuzzy logic, and genetic algorithm.
\end{abstract}

\section{Keywords}

Call Admission Control (CAC), Soft Computing (SC), Artificial Neural Network (ANN), Fuzzy Logic (FL), Genetic Algorithm (GA).

\section{INTRODUCTION}

Basically multimedia networks refer to the transmission of the multimedia information namely voice, video, text, data, graphics and images often at the same time in interactive and possibly real time modes (figure-1). The real-time multimedia services in the multimedia network such as video streaming; video conferencing and Internet Protocol television (IPTV) are experiencing emerging needs in the latest era.

The increasing demand of mobile multimedia application has many requirements in terms of quality of services $(\mathrm{QoS})$. The people always want best connections anywhere and anytime. The advent of $3 \mathrm{G}$ and $4 \mathrm{G}$ services like UMTS and CDMA, cellular networks, Wi-MAX and Mobile Broadband Wireless Access are some of the revolutionary example in mobile communications. The emerging needs for mobile services and internet access shows a number of parallel developments in mobile communications [28]. To satisfy these demands a variety of access technologies are available like wireless fidelity (WiFi), worldwide interoperability for microwave access (Wi-MAX) and cellular networks. It is very difficult for service provide to choose the best network of requesting services and to control the quality level of ongoing connections [10].

Call admission CAC has been widely studied in wireless networks as a crucial tool for QoS provisioning [9]. Quality of service $(\mathrm{QoS})$ is an important tool to measure the efficiency of call admission control in mobile multimedia networks of the signal quality, packet delay, jitter and loss rate, call blocking and dropping probabilities, and transmission rate.

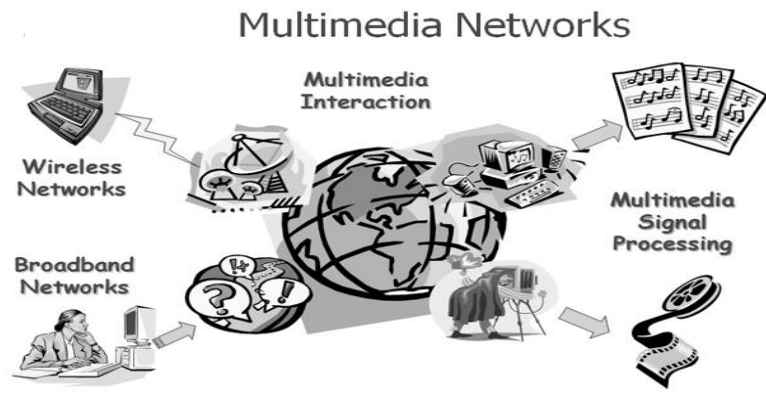

Fig1: Multimedia network.

The main goal of CAC mechanism is to decide whether a new call is to be admitted into the network or not, it is totally depending on network load conditions which could be free or slightly impeded or fully impeded and based on this a decision is made whether to accept / reject the call. This decision effect the QoS of the newly admitted and the existing calls in the network. Hence a new call is admitted if enough idle resources are available in order to meet the QoS requirements for the newly admitted call without violating the QoS for already accepted calls, otherwise the call is rejected [18].

The importance of soft computing in the domain of mobile multimedia networks is separated into the three categories, like optimization, prediction and uncertainty management [16]. The scope of mobile communications extends to three crucial areas, namely: bandwidth, Radio link systems, and cellular mobile communication. These areas of mobile communication form the basis of this review. The components of soft computing are FL, NN and GA. This paper reviews the best feature of soft computing that improves the capability of existing methods of CAC in mobile multimedia networks.

\section{RELATED WORK}

CAC has been widely studied in wireless networks as a crucial tool for QoS provisioning and congestion control. Different reason for CAC has been for design and performance analysis, specially in terms of broadband integrated service digital network (B-ISDN) based on asynchronous transfer mode (ATM) technology have been investigated. But, the main issue of CAC in wireless/mobile networks is more advance due to the special characteristics of wireless/mobile networks such as channel impairments, handoff requirements, channel multiple access interference, and limited bandwidth [9].

In [1], Youssef, I. et al. proposed a novel call admission control algorithm for ATM networks using neural networks. The aim of this paper is to achieve an efficient algorithm that allows for multiplexing gain in terms of processing and 
storage requirements and robustness, so that it could be suitable for all types of traffic in networks. In this paper two objectives are achieved using perceptron and hierarchical model of neural networks.

In [2], I. Khalil et al. proposed a hybrid neural network approache's to estimate cell loss rate of variable bit rates video traffic for CAC in ATM environment. In this approach $\mathrm{NN}$ is used to refine the evaluation results from applying the knowledge gained from the actual performance of the histogram scheme. Performance data which is derived from histogram that can be used to train data onto the NN, which produced better results than the pure histogram based approaches. Through NN the cell loss rate estimation is better than histogram approximations. NN learns to capture the unknown complex relationship between the past and future values of the connection to provide estimation of the cell lose rate in different traffic situation and this learning process is done offline. Since the cell loses processes would be very busty and the request cell lose rate is very small in ATM networks, it's very difficult to estimate CLR from a limited number of training patterns.

In [3] C. Chang et al. proposed a neural fuzzy control for radio resource management in hierarchical cellular systems support in multimedia services. A neural fuzzy resource manager mainly contains a neural fuzzy channel allocation processor (NFCAP). Generally NFCAP is two layered architecture. A fuzzy cell selector is used in the first layer and a neuro fuzzy call admission and rate control is used in second layer. The NFRM use the learning capabilities of the neural networks to reduce the decision error of these conventional channel assignment schemes resulting from modeling.

In [4], YOUSSEF, I. et al. proposed a new neuro computing algorithm for Asynchronous Transfer Mode (ATM) networks. The proposed algorithm applied neural networks to calculate the required bandwidth to support multimedia traffic with multiple QoS requirements. The NN controller calculate the bandwidth per cell using online measurements of the traffic via its count process instead of depending on simple parameters such as burst length, peak, and average bit rate.

In this paper artificial neural network (ANN) is used to maintain dynamically resource shearing between Book-Ahead (BA) and instantaneous request (IR) that produce the high preemption rate of ongoing IR calls. The ANN model measures the network load parameters and appropriate level of preemption rate into appropriate tuning parameter. Once the model is trained it automatically estimate the tuning parameter value essential to achieve the desired level of preemption rate. The simulation result clearly identified that the model closely matche's with the target rate and reduce the preemption rate of on-going IR calls [5].

The total number of available channels is divided into two parts; one is reserved for new admitted calls and second is reserved for handoff calls. A call admission control problem is discussed from computational point of view. Developing various mathematical approaches to solving the call admission control problem is described for the usage of Markov decision process, fuzzy logic, neural networks and genetic algorithms [6].

In [7], Atsushi-Hiramatsu proposed an adaptive call admission control using neural networks for the ATM communications networks. In this paper the author integrate adaptive call admission control and adaptive link capacity control that provides an efficient ATM traffic control system appropriate for multimedia communication services with unknown traffic characteristics. Further, adaptive link capacity control using neural networks that trained to estimate the call loss rate from link capacity that is optimized by a random optimization method according to the estimated call loss rate and observed traffic.

In [8], K. Piamrat et al proposed an admission control system based on Quality of Experience (QoE) gain by users. The human QoE is acquired by a tool called Pseudo Subjective Quality Assessment (PSQA), which is developed with the help of statistic learning using Random Neural Network (RNN). An alternative relying on technical parameters such as latency and bandwidth, which do not have any connection with human knowledge. The proposed scheme based on Mean Opinion Score (MOS) but without interaction from real humans.

In [13], Rezgui et al. proposed a stochastic distributed admission control scheme (so-called RCAC) for WMNs, which uses an analytical model to compute the appropriate admission ratio that guarantees that the loss rate in the network does not exceed a target value and to compute the end-to-end delay that is necessary to process the flow requests based on some delay constraints. However, the cases of link failure and dynamic channel allocation are not considered in the design of this scheme. In addition, this scheme does not consider the case of interflow interference, paying no heed to the contending flows. Whenever a flow is initiated, it has an adverse effect on the capacity of the nodes in the neighboring B-cliques.

In [14], Ramesh Babu H.S. et al. proposed a fuzzy neural approach for call admission control in multi class traffic based Next Generation Wireless Networks (NGWN). They proposed Fuzzy Neural Call Admission Control (FNCAC) scheme is an integrated CAC module that combines the linguistic control Capabilities of the fuzzy logic controller and the learning capabilities of the neural networks .The model is based on Recurrent Radial Basis Function Networks (RRBFN) which has better learning and adaptability to develop the intelligent system to handle the incoming traffic in the heterogeneous network environment. The proposed FNCAC can be achieving by reduced call blocking probability and keeping the resource utilization at an optimal level.

In [15], Zhang et al. proposed a joint admission control and routing protocol that provides QoS guarantee in WMNs based on IEEE 802.16. The problem is designed as a linear Markov decision process and solved using the policy iteration algorithm, the value iteration algorithm, combined with linear programming optimization techniques. In these solution approaches multiple service classes are prioritized by imposing a different reward rate for each service class, yielding maximized network revenue while guarantee the connection blocking probabilities of higher priority service or handoff connections. However, this scheme does not treat the case where co-channel interference and packet-level QoS (such as packet delay) are considered as design parameters of the admission control and routing problem in WMNs.

The computation intelligence based CAC use evolutionary approaches' like Genetic Algorithm (GA), fuzzy logic and Artificial Neural Networks (ANN). Majority of the computational intelligence based CAC algorithms incorporate fuzzy logic, fuzzy neural and fuzzy MCDM methods. There are very few works reported on the usage of Artificial Neural Networks in CAC and Genetic algorithms are the most popular evolutionary algorithm [10]. 
In [11] J.D. Mallapur et al. proposed a Fuzzy based bandwidth allocation scheme, assume that the multimedia application can tolerate transient functions in the QoS and allows for temporary borrowing of bandwidth from existing connections in order to accommodate newly arrival call connections. Some of the users may move out of the cell thus releasing the allocated bandwidth for specific users. The same can be redistributed with minimal disturbance to all of the existing users because of the highly time varying nature of the traffic, sudden burst and simultaneously arrive leading to serious over loading of the network resources.

In [22] Nordstrom et al. proposed three ANN models for the CAC in ATM networks. It is used for the implementation of link QoS formula, based on the heterogeneous fluid flow queuing model. The first model based predefined peak rate parameters, whereas the second model is based on a state interpretation of aggregated link traffic, and the third model based on a statistical pre-processing of the traffic parameters [23]. These models help in computation of accurate cell loss in the heterogeneous fluid flow model.

A neuro-dynamic programming which includes decomposition is used, in which neuro-dynamic programming and reinforcement learning, along with a decomposition methodology to develop a dynamic (state-dependent) CAC and routing policies has been introduced. These policies in this paper mainly depend on the state dependent link costs followed by a simulation based learning method which is employed to tune up the parameters that define the link costs. A range of experiments indicates the robustness of the proposed policy and the performance is compared with a commonly used heuristic [24].

In [34] Shuaibu et al. propound fuzzy logic based CAC. The mechanism involves partitioning of the total bandwidth into three constant bit rate, variable bit rate and handover (HO). FL mainly deals with the HO partition so as to keep the probability of call dropping as low as possible for the available bandwidth. The simulation results shows that FL based CAC performs better when compared to Partition based $\mathrm{CAC}$ and Conventional Bandwidth based CAC.

In [35] Yufeng et al. present A fuzzy CAC scheme, which guarantees the quality of service provision in wireless networks by searching automatically the optimal number of guard channels in a base station for effective utilization of resource. Simulation results show that the proposed fuzzy CAC performs better by increasing the resource utilization of the network. The fuzzy scheme has better robust performance when compared to adaptive channel reservation.

The increasing demand of efficient bandwidth utilization in terms of resource availability is directly proportional to the increased demand of advanced network services such as video and data. One of the main techniques for supporting the data and video services is dynamic channel allocation along with QoS parameters.

In [22] Nordström et al. proposed a dynamic channel allocation (DCA) method used to avoid traffic modeling for audio/ video conferences. This method is used to combine the advance idea of genetic algorithms for time management and predicting exact value and results. The main objective of this idea is that it ensures High QOS to wireless Audio/video conference users.

\section{CALL ADMISSION CONTROL SCHEMES}

The main work of CAC schemes is to decide whether a call should be admitted by the network or not. In mobile multimedia network CAC scheme has been proposed to control the packet dropping rate for video conference services to maintain the QoS. When a call admission is requested, this represents a possible gain to the network profits because of the expected reward due to the resource utilization. Meanwhile, the incoming call might cause congestion and degradation in the resources allocated to the already admitted calls, which in turn can cause a reduction in the network profits. Some CAC schemes are described in the following table [29].

The admissible region can be used for call admission by examining the network state to determine whether it is inside the admissible region. It should be noted that the admissible region depends on the used scheduling scheme.

Table 1: Call admission control schemes

\begin{tabular}{|l|l|}
\hline $\begin{array}{l}\text { CAC for } \\
\text { Transmissi } \\
\text { on and Rate } \\
\text { Control }\end{array}$ & $\begin{array}{l}\text { CAC schemes can ensure a minimum } \\
\text { transmission rate either limiting the network } \\
\text { load by minimizing the transmission rate } \\
\text { degradation }\end{array}$ \\
\hline $\begin{array}{l}\text { CAC for } \\
\text { Controlling } \\
\text { Packet } \\
\text { level }\end{array}$ & $\begin{array}{l}\text { CAC scheme estimates the packet delay and } \\
\text { delay jitter in multiple class wireless ATM } \\
\text { networks. If packet delay and delay jitter can } \\
\text { be guaranteed, the calls are admitted } \\
\text { otherwise, it is rejected. }\end{array}$ \\
\hline $\begin{array}{l}\text { Revenue } \\
\text { based CAC } \\
\text { Schemes }\end{array}$ & $\begin{array}{l}\text { CAC scheme maximizes the revenue by } \\
\text { minimizing the penalty incurred by dropping } \\
\text { an existing call and carrying out a channel } \\
\text { reassignment. }\end{array}$ \\
\hline $\begin{array}{l}\text { CAC for } \\
\text { Service/ }\end{array}$ & $\begin{array}{l}\text { Calls are admitted with three possible } \\
\text { prioritization schemes. The first scheme gives } \\
\text { higher priority to voice service than that of } \\
\text { data service by using a higher power level } \\
\text { threshold. Conversely, The second scheme } \\
\text { gives higher priority to data service than that } \\
\text { of voice service by using a higher power level } \\
\text { threshold to prioritize the data service. Finally, } \\
\text { the third scheme uses the same threshold for } \\
\text { both services. }\end{array}$ \\
\hline $\begin{array}{l}\text { CAC for } \\
\text { Fairness }\end{array}$ & $\begin{array}{l}\text { CAC scheme uses virtual partitioning with } \\
\text { priority (VPP). In VPP the priority of each } \\
\text { class is reduced if this class is overloaded. }\end{array}$ \\
\hline
\end{tabular}

\section{SOFT COMPUTING TECHNIQUES}

Soft computing is a collection of computational techniques in computer science used to solve the real world problems introduced by Lotfi A. Zadeh [17]. It is an effective complement of conventional artificial intelligence in the domain of computational intelligence that is used to solve the problems whose solutions are represented in partial truth and approximation form rather than an exact form. In retrospect, the idea of extended soft computing was proposed in [25] by incorporating immune network theory and chaos Computing. In the same vein, Fuzzy logic, which is the foundation of soft computing, has been expanded [26]. 
The main objective of soft computing is to utilize computation, reasoning and inference to reduce computational cost by exploiting tolerance for inaccurate, unpredictable, partial truth and approximation. The components of SC are FL, ANN, and GA. Every component of the SC has their own ability to compute while solving the problems i.e. uncertainty management for incomplete modeling and Insufficient measurement are handled by FL, Prediction tasks are handled by ANN, Optimization is handled by evolutionary algorithms and though genetic algorithms are somewhat separated from the other two, they can effectively perform the role of a trainer for both neural networks and fuzzy systems.

\subsection{Artificial Neural Network}

Neural networks were first introduced by Mc Culloch Pitts in 1943 and Artificial Neural Networks (ANN) is inspired from biological neurons. An ANN consists of small computing units, called neurons, arranged in different layers and interconnected with each other with some nerves called synapses. These neurons calculate the results from the inputs which are derived from the network e.g. if a network topology is to be setup, all available inputs specific to topology are given into the neurons [27].

The Processing of Information take place at many simple processing elements called nodes (also known as cells, units or neurons). Each link between nodes is associated with a weight, which controls the behavior of the trained ANN. The main advantages of ANN are distributed memory, parallelism, generalization capability, learning and redundancy. However, ANNs have some drawbacks that need to be complemented by other techniques. Neural networks are "black box" in nature; they do not provide the meaning of weight changes that gives a particular input-output, relationship. Additionally, it is challenging to determine the proper size and structure of an ANN to solve a particular problem [28].

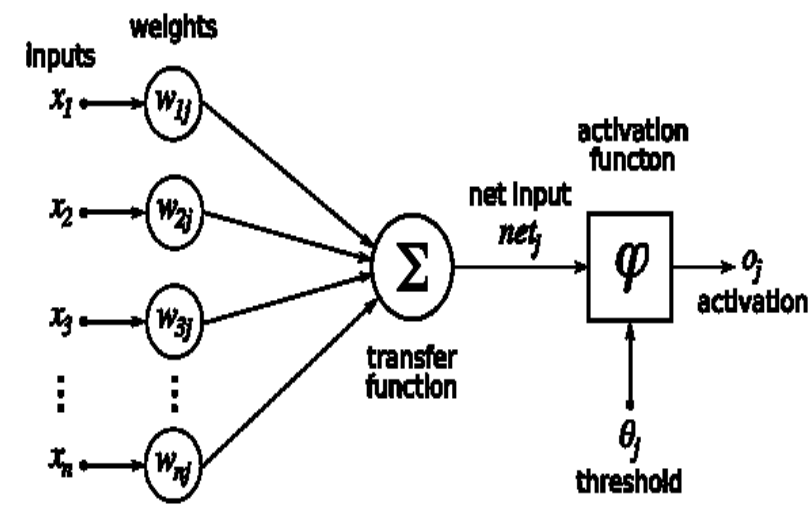

Fig 2:- Structure of an artificial neuron network [27]

Now-a-days the intelligent techniques have been widely applied to nonlinear time varying and complicated problems that were challenging [3]. ANNs are universal approximators capable of approximating any nonlinear function and used to control, prediction and classification of any problem by using complex nonlinear functions. Thus a single implementation of ANN can be used to model several scenarios. ANNs also help offset the computational cost associated with complex mathematical and electromagnetic analysis.

In CAC, ANN is used to reduce the call Blocking / dropping probability by the neural network model where the required neuron are activated and produce end results. On the basis of the final result, the decision is made for the acceptance or rejection of the calls based on available bandwidth [18].

\subsection{A Fuzzy Logic}

Fuzzy logic (FL) is a methodology for representing uncertain and imprecise knowledge, developed by Zadeh [9]. FL is used to solve problem whose solutions are presented in the form of range rather than in the form of true or false. Fuzzy set can be defined as an extension of the ordinary set, called a crisp set, every element of the fuzzy set assigned a membership value between I and 0 that shows the belongingness to this fuzzy set.

The main benefit of FL approach [12] are, easy to understand and build a predictor for any desired accuracy with a simple set of Fuzzy rules. Fuzzy logic offers simplicity, flexibility and cost effectiveness. Due to less computational demand there is no need of mathematical model for estimation of future values. When dealing with mobile multimedia networks, it is impractical to obtain a comprehensive knowledge of all factors. This emphasizes the need for a method or means of tracking uncertainty and ambiguity. Fuzzy logic uses human expert knowledge in a rule based framework for solving a variety of problems in wireless and mobile networks [28].

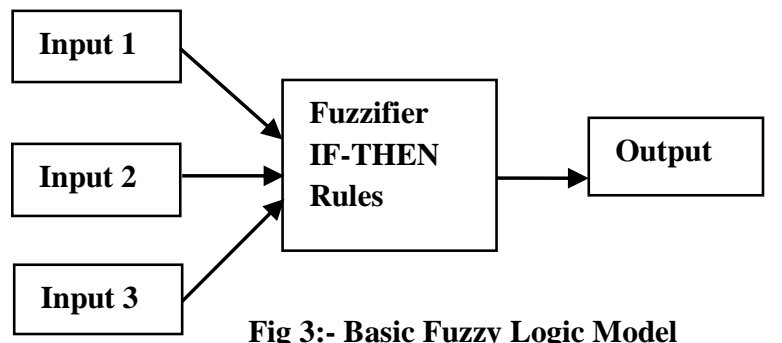

In CAC FL is used for decision making when a new call or handoff call request is received. FL is also used to predict the future location of the user on the basis of location management [19].

\subsection{Genetic Algorithm}

Genetic algorithms (GA's) combine the rules of natural selection and genetics to form a search algorithm for optimization problems [15]. GA's is slightly different from the traditional search algorithms, in the form of their ability to search for the optimum is not confided to any particular type of constraint. GA is used to solve the problem by using process of natural evolution methods like inheritance, mutation, selection and crossover. The input data are collected and sent to the GA, to obtain the end result that can be derived in different ways like inheritance and mutation. In inheritance the input data will have direct impact on the end result as it has strong connection with it and in mutation sudden changes are calculated to arrive at end results. Later in crossover two or more results are crossover or exchanged to arrive at the end result. In selection process, only the right one or correct one is selected to obtain a final result. By combining this entire process, the $\mathrm{NN}$ solves the problem in a faster rate compare to other methods [20]. 


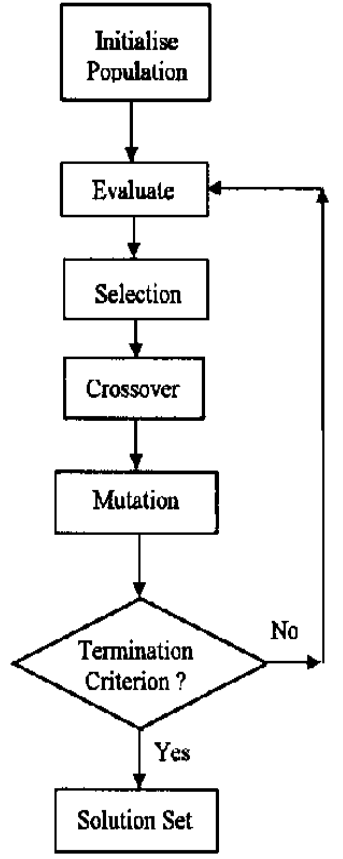

Fig 4: Basic Architecture of Genetic Algorithm

Genetic algorithm has the capability to optimize both continuous and discrete variables and it doesn't require derivative information. Optimization is get more importance in wireless communications according to the increasing complexity of new technologies, parallel operation of different wireless/mobile networks and the pressing need for efficient use of available of spectral and network resources. Genetic algorithm presents itself as a very effective tool for addressing the multi-faceted optimization needs of wireless networks
A GA in CAC plays a major role during the resource reservation for calls requiring guaranteed services. Especially in case of the multimedia call like video, audio and data the ATM networks has its own QoS requirements like cell loss rate, delay, jitter, etc [20].

\section{CONCLUSION AND FUTURE SCOPE}

The paper presents a broad review of soft computing techniques with prime focus on call admission control in mobile multimedia networks. We have compared the bandwidth and link control parameters' performance of existing systems of CAC schemes in mobile multimedia network using soft computing. In most of the soft computing techniques, we found that artificial neural network has been widely used. Therefore we can say that ANN is mostly used. On the other hand fuzzy logic (FL) and genetic algorithm (GA) are least used. Performance estimation results show that the ANN is more accurate in calculating bandwidth. Accordingly, admission control decisions, which are more accurate and the problem of call dropping or rejection can be reduced to a greater extent. So far, back propagation and feed forward neural networks are most commonly used. These methods are supervised learning approaches of ANN. Other ANN methods like delta learning and feedback network can also be used to handle some of the call admission control issues. But, unsupervised learning methods like adaptive resonance theory (ART1), Kohonen's self organizing map (SOM), counter propagation network (CPN) etc. can also be used in future to improve the QoS mechanism which is also suitable for CAC with limited resources/bandwidth; and it has the advantage such as less call blocking and dropping probability, less bandwidth consumption and better resource utilization etc. Consequently we can say that artificial neural networks play a very important role for CAC issues like bandwidth allocation. On the other hand genetic algorithm and fuzzy logic can also be used to optimize the problem of CAC in mobile multimedia networks with respect to quality index and decision maker.

Table 2: Results: Based On Bandwidth

\begin{tabular}{|c|c|c|}
\hline & Capacity & Accuracy \\
\hline \multicolumn{3}{|l|}{ Neural Network for ATM network } \\
\hline $\begin{array}{l}\text { Compute the effective bandwidth required for } \\
\text { variable bit rate video traffic. }\end{array}$ & $\begin{array}{l}\text { Single link with maximum capacity } \\
155 \mathrm{Mb} / \mathrm{s} \text {. }\end{array}$ & $\begin{array}{l}\text { It can make fast decision in bandwidth } \\
\text { allocation due to high computation rates } \\
\text { with a great accuracy [2]. }\end{array}$ \\
\hline \multicolumn{3}{|c|}{ Neural Network for bandwidth allocation in ATM networks } \\
\hline $\begin{array}{l}\text { Calculate the required bandwidth with multiple } \\
\text { QoS. }\end{array}$ & $\begin{array}{l}\text { Total link capacity } 155 \mathrm{Mb} / \mathrm{s} \text { after } \\
\text { approximately } 600 \text { frames error is } \\
\text { less than } 2 \% \text {. }\end{array}$ & $\begin{array}{l}\text { IDC is used as a measurement of accuracy } \\
\text { which provides more accurate results [4]. }\end{array}$ \\
\hline \multicolumn{3}{|c|}{ Neural Fuzzy Resource Manager for Hierarchical Cellular Systems } \\
\hline $\begin{array}{l}\text { Neural Fuzzy Network for cellular network used } \\
\text { to perform radio resource management. }\end{array}$ & $\begin{array}{l}\text { The NFRM gains } 31.1 \%, 6.3 \% \text {, } \\
21.4 \% \text { improved over OCA, CCA } \\
\text { and FCAC methods. }\end{array}$ & More Accurate [3]. \\
\hline \multicolumn{3}{|c|}{ Intelligent Model to Control Preemption Rate in Networks with Book- Ahead Reservation } \\
\hline $\begin{array}{l}\text { Preemption rate of on-going calls in a QoS } \\
\text { enabled networks. }\end{array}$ & $\begin{array}{l}\text { Very low preemption rate causes } \\
\text { low utilization of network capacity. }\end{array}$ & $\begin{array}{l}\text { Training algorithms SCR \&BR provide } \\
\text { mean error } 2.51 \% \& 1.88 \% \text { respectively [5] }\end{array}$ \\
\hline
\end{tabular}


Call Admission Control and Link Capacity Control by Distributed Neural Networks in integrated neural network.
Link capacity optimization is created by on-line training with back propagation.
After training the root mean square errors over 1000 random points the calculated error over ten trials with different initial weight values [7].

QoE-aware Admission Control for Multimedia Applications in IEEE 802.11 Wireless Networks

CAC mechanism based on quality of experience using random neural networks.
Scheme will grant all network capacity to a small number of flows for high threshold.
This approach provides high accuracy but at high costly and time consuming [8].

Neural Decision Making for Decentralized pricing structure of a telecommunication network

An original decision making process is studied

to decentralize the proposed CAC mechanism.

Link capacity for decentralized

telecommunication network is 30 Mbps.
Neural approach provides fast decision making capability with high accurate results [12].

Intelligent Schemes for Bandwidth Allocation in Cellular Mobile Networks

Bandwidth allocation schemes for cellular mobile networks namely using Back

Propagation Neural Network (BPN) and Particle Swarm Optimization (PSO).

\section{REFERENCES}

[1] S. Youssef, I. Haibib, T. Saadawi. 1995. ATM Call Admission Control Using Neural Networks" 0-78032489- 7195 IEEE.

[2] I. Khalil, B. Mohd Ali. 1995. A Hybrid Histogram and Neural based Call Admission Control for VBR Video Traffic. 'Artificial Neural Networks 'conference Publication No. 409.

[3] K. Lo, C. Chang, C. Shung, 2003. A Neural Fuzzy Resource Manager for Hierarchical Cellular Systems Supporting Multimedia Services" IEEE Transmission on Vehicular technology, vol. 52.

[4] Youssef, I. Haibib, T. Saadawi. 1997. A Neuro computing Controller for Bandwidth Allocation in ATM Networks. IEEE journal on selected areas in communications, vol. 15 , no. 2 .

[5] I. Ahmad, J. Kamruzzaman, D. Habibi,F. Islam. 2008. An Intelligent Model to Control Preemption Rate of Instantaneous Request Calls in Networks with BookAhead Reservation, 978-1-4244-2603-4/08 IEEE.

[6] J. GoriP, C. DoboS, A. Ciimhr. 2003. Computational Intelligence in Call Admission Control. 0-7803-79934/03 lEEE.

[7] Atsushi -Hiramatsu. 1991. Integration of ATM Call Admission Control and Link Capacity Control by Distributed Neural Networks" IEEE journal on selected areas in communications, vol. 9 , no. 7 .

[8] K. Piamrat, A. Viho, J. Bonnin. 2008. QoE-aware Admission Control for Multimedia Applications in IEEE 802.11 Wireless Networks, 978-1-4244-1722 IEEE.

[9] M.ahamed.2005. Call Admission Control in Wireless Network: A Comprehensive Survey. volume 7, no. 1, IEEE communication.

[10] P. Metre, K. Radhika, Gowrishankar. 2012. Survey of Soft Computing Techniques for Joint Radio Resource Management, 978-1-4673-1520, IEEE

[11] J.D. Mallapur, S. Abidhusain, S. S. Vastrad, A. C. Katageri. 2010. Fuzzy Based bandwidth Management for
Minimizing bandwidth allocation for individual users [30]. Wireless Multimedia Networks" CCIS 70, pp. 81-90, Springer-Verlag Berlin Heidelberg.

[12] F. Davoli, M. Marchese, M. Mongelli. 2005. Neural Decision Making for Decentralized Pricing-based Call Admission Control, 0-7803-8938- IEEE.

[13] J. Rezgui, A. Hafid, M. Gendreau. 2008. A distributed Admission control scheme for wireless mesh networks, In Proc. 5th Int. Conf. broadnets, London, U.K. pp. 594 601.

[14] Ramesh Babu H.S. Gowrishankar, Satyanarayana P.S. 2010. An Intelligent Call Admission Control Decision Mechanish for Wireless Networks journal of computing, volume 2, issue 4, issn 2151-9617.

[15] S. Zhang, F. R. Yu, V. C. M. Leung. 2010. Joint connection admission control and routing in IEEE 802.16-based mesh networks.IEEE Trans. Wireless Commun., vol. 9, no. 4, pp. 1370-1379.

[16] Matthew K. Luka, Aderemi A. Atayero. 2012. Applications of Soft Computing in Mobile and Wireless Communications", Covenant University,Ota, Nigeria, International Journal of Computer Applications (0975 8887) Volume 45- No.22.

[17] Lotfi A. Zadeh. Fuzzy Logic, Neural Networks and Soft Computing", Communications of the ACM, Vol. 37.

[18] Mahesh G, Yeshwanth S, Manikantan U V. 2014. Survey on Soft Computing based Call Admission Control in Wireless Networks, International Journal of Computer Science and Information Technologies, Vol. 5 (3), 3176 -3180 .

[19] BAE, I-H, OH, S-J, Olariu, S. 2003. A fuzzy-logic Based location management method for mobile Network, in Intl. Conf. software engineering research And application SERA pp 304-319.

[20] Sherif, Mahmoud R., Ibrahim W. Habib, Mahmoud Nagshineh, Parviz Kermani. 2000. Adaptive Allocation of Resources and Call Admission Control for Wireless Atm Using Genetic Algorithms. Selected Areas in Communications, IEEE Journal on 18, No. 2.

[21] Peter Marbach, Oliver Mihatsch, and John N. Tsitsiklis. 2000. Call Admission Control and Routing in Integrated 
Services Networks Using Neuro-Dynamic Programming, IEEE journal on selected areas in Communications, vol. 18 , no. 2.

[22] Nordström, Ernst, Olle Gallmo, Lars Asplund, Mats Gustafsson, Bo Eriksson. 1992. Neural networks for admission control in an ATM network. In Connectionism in a broad perspective: Selected papers from the Swedish Conference on Connectionism, pp. 239-250.

[23] Dakroury, Yasser, Ahmed Abd, Osman Badr. 1999. Testing the performance of a neural networks-based adaptive call admission controller with multimedia traffic, In Multiple Approaches to Intelligent Systems, pp. 80-91. Springer Berlin Heidelberg.

[24] Marbach, Peter, Oliver Mihatsch, John N. Tsitsiklis. 2000. Call admission control and routing in integrated services networks using neuro-dynamic programming Selected Areas in Communications, IEEE Journal, VOL. 18, No. 2, page: 197-208.

[25] Taniguchi S., Dote Y, Ovaska S.J. 2000 .Control of intelligent agent systems (robots) using extended soft computing, IEEE Int'l conf. on Systems, man and cybernetics vol.1, pp. 3568-3572.

[26] Q. Liang,J.M. Mendel. 2000. Interval Type-2 Fuzzy Logic Systems: Theory and Design, IEEE Tran. On Fuzzy Systems vol.8, pp. 535-550.

[27] N O. Hayking. 2008. Neural Networks and Learning Machines" (3rd Edition), Published By Prentice Hall, ISBN-13: 978-0131471399 ISBN-10: 0131471392.

[28] Aderemi A. Atayero ,Matthew.K. Luka. 2012. Applications of Soft Computing in Mobile and Wireless Communications, International Journal of Computer Applications (0975 - 8887) Volume 45-No.22.

[29] Georgios I. Tsiropoulos, Dimitrios G. Stratogiannis, Eirini Eleni Tsiropoulou.2010.Call Admission Control in Mobile and Wireless Networks, Mobile and Wireless Communications Network Layer and Circuit Level Design, Salma Ait Fares and Fumiyuki Adachi (Ed.), Isbn: 978-953-307-042-1, Intech.
[30] Isvarya Luckshmi, A.P., Visalakshi, P. Karthikeyan, N.K. 2011.Intelligent Schemes for Bandwidth Allocation in Cellular Mobile Networks, international Conference on Process Automation, Control and Computing (PACC) pp. 1-6.

[31] K. Kumar, R. Singh, Z. Khan, A. Indian. 2008. Air traffic runway allocation problem artmap (art1), Published in Ubiquitous Computing and Communication Journal, Korea, ISSN: 1992-8424, Vol 3, No 3.

[32] K. Kumar, R. Singh, Z. Khan. 2008. Air traffic enroute conflict detection using adaptive resonance theory map neural networks (art1), Published in Ubiquitous Computing and Communication Journal, Korea, ISSN: 1992-8424, Vol 3, No 3.

[33] Krishan Kumar. 2012. ART1 Neural Networks for Air Space Sectoring". International Journal of Computer Applications 37(1):20-24, ISSN: 0975-8887. Published by Foundation of Computer Science, New York, USA.

[34] Shuaibu, D. S., S. K. Yusof, N. Fisal, S. H. S. Ariffin, R. A. Rashid, N. M. Latiff, Y. S. Baguda. 2011. Fuzzy Logic Partition-Based Call Admission Control for Mobile Wimax, Isrn Communications And Networking, No 16 .

[35] Y. Ma, X. Hu, Y. Zhang, Y.Shi. 2005. Intelligent Call Admission Control Using Fuzzy Logic In Wireless Networks, In Proceedings Of The American Control Conference.

[36] Sathyapriya R, S. Malathy.2012. Dynamic Channel Allocation and Revenue Based Call Admission Control Using Genetic Algorithm, International Journal of Advanced Research in IT and Engineering, ISSN: 2278-6244, Vol.1, pp 33- 46.

[37] Jacek M Zurada.1992. Introduction to artificial neural systems, west publishing company St. Paul New York Los Angeles San Francisco. 